\title{
Effects of intrinsic aging and photodamage on skin dyspigmentation: an explorative study
}

\author{
Gabor Dobos \\ Carina Trojahn \\ Brian D'Alessandro \\ Sachin Patwardhan \\ Douglas Canfield \\ Ulrike Blume-Peytavi \\ Jan Kottner
}




\title{
Effects of intrinsic aging and photodamage on skin dyspigmentation: an explorative study
}

\author{
Gabor Dobos, ${ }^{\mathrm{a}, \star}$ Carina Trojahn, ${ }^{\mathrm{a}}$ Brian D'Alessandro, ${ }^{\mathrm{b}}$ Sachin Patwardhan,, Douglas Canfield, ${ }^{\mathrm{b}}$ \\ Ulrike Blume-Peytavi, ${ }^{a}$ and Jan Kottner ${ }^{\mathrm{a}}$ \\ ${ }^{a}$ Charité-Universitätsmedizin Berlin, Clinical Research Center for Hair and Skin Science, Department of Dermatology and Allergy, \\ Charitéplatz 1, 10117 Berlin, Germany \\ ${ }^{\mathrm{b} C}$ Canfield Scientific Inc., 4 Wood Hollow Road, Parsippany, New Jersey 07054, United States
}

\begin{abstract}
Photoaging is associated with increasing pigmentary heterogeneity and darkening of skin color. However, little is known about age-related changes in skin pigmentation on sun-protected areas. The aim of this explorative study was to measure skin color and dyspigmentation using image processing and to evaluate the reliability of these parameters. Twenty-four volunteers of three age-groups were included in this explorative study. Measurements were conducted at sun-exposed and sun-protected areas. Overall skin-color estimates were similar among age groups. The hyper- and hypopigmentation indices differed significantly by age groups and their correlations with age ranged between 0.61 and 0.74 . Dorsal forearm skin differed from the other investigational areas $(p<0.001)$. We observed an increase in dyspigmentation at all skin areas, including sunprotected skin areas, already in young adulthood. Associations between age and dyspigmentation estimates were higher compared to color parameters. All color and dyspigmentation estimates showed high reliability. Dyspigmentation parameters seem to be better biomarkers for UV damage than the overall color measurements. (C) 2016 Society of Photo-Optical Instrumentation Engineers (SPIE) [DOI: 10.1117/1.JBO.21.6.066016]
\end{abstract}

Keywords: skin aging; melanin; dyspigmentation; hyperpigmentation; hypopigmentation; reliability.

Paper 160112R received Feb. 24, 2016; accepted for publication May 25, 2016; published online Jun. 22, 2016.

\section{Introduction}

The world population is growing and aging and skin diseases of the elderly have become an increasing concern globally. ${ }^{1}$ Age-related skin changes lead to a higher vulnerability to various external insults. ${ }^{2}$ Skin aging is influenced by genetic predisposition and the passing of time called intrinsic aging. Environmental factors such as sun exposure, drug intake, and smoking can accelerate skin aging. This is frequently referred to as extrinsic aging or photodamage. ${ }^{3,4}$ Clinical signs of intrinsic aging are benign lesions (e.g., seborrheic keratosis), fine wrinkling, lax appearance, and subcutaneous fat atrophy. Extrinsic aging results in coarse wrinkles, elastosis, pigmentary changes, and skin malignancies. ${ }^{2-5}$

The two major chromophores of the skin pigment system are melanin and hemoglobin. Melanin serves as a protective barrier against carcinogenesis. It absorbs UV radiation that leads to DNA and protein damage. Thus, lighter skin types are more prone to develop skin cancer. ${ }^{2,6,7}$ However, the skin melanin system itself is also altered by aging. This leads to the appearance of hyperpigmented lesions such as freckles or solar lentigines and hypopigmented changes, e.g., pseudoscars. ${ }^{8}$ Various forms frequently accompany each other. These pigmentary heterogeneities together are frequently called dyspigmentation. ${ }^{6,9}$

Increasing amount of hemoglobin in the skin leads to erythema. Recently, distinct subtypes of facial skin aging have been described, characterized by an increase of facial erythema, distinct of rosacea. ${ }^{10,11}$ Whether this occurs also on other sun-protected areas is not known.

\footnotetext{
*Address all correspondence to: Gabor Dobos, E-mail: gabor.dobos@
} charite.de
Various instrumental methods exist to quantify skin pigmentation. Skin color is frequently measured by reflectance spectrometric techniques. Erythema indices and $a *$ values from the CIE $L * a * b *$ color space are used to quantify redness. $L * b *$, and melanin indices are used to measure the brown pigmentation. Facial skin was shown to become darker with increasing age ${ }^{12-14}$ and to exhibit more erythema in certain cases. $^{10,11}$

Quantification of skin dyspigmentation is in its infancy. In addition to changes of overall pigmentation intensity, empirical evidence indicates an age-related increase of facial dyspigmentation in Caucasian ${ }^{15-18}$ and Asian ${ }^{19}$ skin types.

Measuring skin dyspigmentation on nonfacial skin areas is clinically relevant to estimate the amount of photodamage and melanoma risk. ${ }^{20-22}$ At the same time, dyspigmentation influences the perceived chronological age. ${ }^{18}$

For a better understanding of photocarcinogenesis and the concept of "sun damage," intrinsic skin aging is frequently compared to photodamaged skin. ${ }^{22}$ To reduce interindividual variations, many clinical trials compare photo-aged and sun-protected skin areas within the same individuals. ${ }^{23}$ Sun-protected areas such as the buttock skin, ${ }^{24}$ the upper inner arm (UIA), ${ }^{25}$ and volar forearm (VFA) ${ }^{26}$ served in clinical trials as a negative control to extrinsic aging. However, one might argue whether all these areas are equally sun protected.

The aim of this study was to quantify and to compare (dys) pigmentation on sun-exposed dorsal forearm (DFA) with the sun-protected areas of the VFA and the UIA. We also aimed to describe the reliability of the obtained parameters.

$1083-3668 / 2016 / \$ 25.00$ ๑ 2016 SPIE 


\section{Methods}

\subsection{Study Design and Setting}

The study was performed at the Department of Dermatology and Allergy, Charité-Universitätsmedizin Berlin $\left(52.3^{\circ} \mathrm{N}, 13.2^{\circ} \mathrm{E}\right)$ in the Clinical Research Center for Hair and Skin Science. Female volunteers of three distinct age groups were recruited for this explorative study between January and April 2014. All subjects gave their written informed consent prior to the visit and the study was conducted in accordance with the principles of the current version of the declaration of Helsinki.

\subsection{Sample}

Skin healthy volunteers were enrolled to have eight subjects in each of three age groups: 30 to 40,50 to 60 , and 70 to 80 years. The chosen sample was considered to display different signs of aging because chronological age is the main predictor for skin aging. ${ }^{27}$ Inclusion criteria were among others no cosmetic or rejuvenation procedures 10 years prior to the study, no hormonal replacement therapy in the past two years, no extreme UV exposure eight weeks before the visit, and nonsmoking status. Subjects with a history of certain medications (e.g., photosensitizing agents, systemic, or topical corticosteroids) or a history of dermatologic diseases were not included in the study. Subjects were asked to avoid water contact at the measurement sites $12 \mathrm{~h}$ prior to the measurements and not to apply any cosmetic products on their arms. All the measurements were noninvasive and were performed in similar and standardized conditions.

\subsection{Measurement Sites}

The measurement fields had a size of $10 \mathrm{~cm} \times 5 \mathrm{~cm}$ and were located parallel to the long bones of the right upper extremity. On the DFA and VFA, the measurement fields were located halfway between the wrist and the cubital lines. On the UIA, it was halfway between the cubital line and the anterior axillar line.

\subsection{Skin-Color Measurements}

Skin color was measured by reflectance spectrophotometric devices. The melanin- and erythema indices were obtained using the Mexameter MX18 (Courage \& Khazaka, Cologne, Germany). This instrument uses narrow-band illumination on the skin at 568, 660, and $880 \mathrm{~nm}$ and parameters are based on the measured reflections. ${ }^{28} L * a *$, and $b *$ from the CIELab color space were measured with the broad spectrum Chromameter CM-700d (Konica Minolta, Osaka, Japan) ${ }^{29}$ at D65 lighting standard. All the color measurements were conducted in triplicate per measurement field. The devices were removed after every single measurement and placed again on the skin surface with a distance of $2 \mathrm{~cm}$.

\subsection{Measurement of Skin Dyspigmentation}

Skin dyspigmentation was quantified by applying an imageprocessing technique described previously. ${ }^{17}$ Standardized clinical images were taken of the measurement fields using a Lab Imaging System (Canfield Scientific, Parsippany, New Jersey) for consistent and standardized lighting; coupled with an arm positioning device. To enhance image quality, the room was completely darkened during photoacquisition. After color calibration, using seven standardized color chips inside the image frame, the "cross-polarized" images were transformed into $\mathrm{RBX}^{\circledR}$-Brown images. ${ }^{30}$ The $\mathrm{RBX}^{\circledR}$ transformation is based on a spectrocolorimetric light transport model of the skin to compute information of melanin (brown) and hemoglobin (red) concentration and distribution. The $\mathrm{RBX}^{\circledR}$ transform is computed from a training database of color-calibrated crosspolarized images. This database is comprised of subjects with various skin types to obtain a unique comparative solution that is independent of the skin type. The $\mathrm{RBX}^{\circledR}$-Brown values are relative to the expected mean pigmentation of the training database population.

The three parameters to quantify skin melanin inhomogeneity based on the $\mathrm{RBX}^{\circledR}$-Brown transformation using image analysis are the hyperpigmentation index,${ }^{30}$ the hypopigmentation index, and the overall pigmentation intensity (Fig. 1). In a first step, the expected average pigmentation values of the areas of interest were calculated. Second, the thresholds were set to define individual areas of hyper- and hypopigmentation, i.e., areas where the melanin concentration is higher and thereby the skin is darker and vice versa. The threshold parameters for this were calculated using the $\mathrm{RBX}^{\circledR}$ training database and were applied after normalizing the image with respect to the subject's individual background pigmentation value. The hyper- and hypopigmentation indices represent the proportion of darker or lighter areas of the entire measurement field relative to the background skin pigmentation of the subject in the $\mathrm{RBX}^{\circledR}$-Brown image. Index values can theoretically range from 0 (no hyper- or hypopigmentation) to 1 (maximum hyper- or hypopigmentation) within the measurement area. The overall pigmentation intensity, negatively correlating with the average melanin concentration, was computed as the average $\mathrm{RBX}^{\circledR}$-Brown value within the measurement area. This value can theoretically range from -25.5 to 26.4 , representing dark to fair skin, respectively, although the typical range for human skin tone is much narrower.

Standardized clinical images were taken twice from the same measurement fields with $3 \mathrm{~h}$ in between to be able to investigate the reliability of the dyspigmentation parameters. Statistical analysis was conducted using the means of both measurements.

\subsection{Statistical Analysis}

Data were analyzed descriptively by calculating means and standard deviations for each measurement per skin area for the whole sample and each group. Comparison of means between the age groups was conducted by one-way analysis of variance (ANOVA). An alpha-value of 0.05 (two sided) was used. Pearson's correlation coefficients were calculated to estimate the association between pigmentation parameters and age. An $r$ smaller than -0.3 or higher than 0.3 was regarded as minimal strength of association.

To compare skin-color measurements and dyspigmentation indices between the measurement fields, paired sample $t$-tests were calculated, using an alpha-value of 0.05 (two sided).

To investigate the reliability of repeated measurements, intraclass correlation coefficients [ICCs $(1,1)]$ were calculated for each variable within repeated measurements per field. Indices greater than 0.7 were considered as good and above 0.9 as excellent test-retest reliability. ${ }^{31,32}$ Statistical analysis was performed using SPSS Version 20. 
Table 1 Sample characteristics.

\begin{tabular}{lcccc}
\hline & Young group $(n=8)$ & Midaged group $(n=8)$ & Old group $(n=8)$ & Total $(n=24)$ \\
\hline Age in years, mean (SD) & $33.5(2.1)$ & $55.4(2.7)$ & $76.6(1.9)$ & 74 to 79 \\
Range & 31 to 37 & 51 to 59 & $26.2(2.5)$ & 31 to 79 \\
BMI in $\mathrm{kg} / \mathrm{m}^{2}$, mean (SD) & $21.8(2.0)$ & $26.0(5.0)$ & 21.1 to 29.3 & $24.7(3.9)$ \\
Range & 19.7 to 25.9 & 20.8 to 30.5 & & \\
Photo type, $n$ & & & 4 & 19.7 to 30.5 \\
II & 4 & 7 & 4 & 9 \\
III & 4 & 1 &
\end{tabular}

Note: SD, standard deviation; BMI, body mass index.

\section{Results}

\subsection{Study Sample}

Sample characteristics are displayed in Table 1. Fitzpatrick photo types and body mass indices of the subjects were comparable. Mean age of subjects was 33.5 years in the young, 55.4 years in the midaged, and 76.6 years in the older group.

\subsection{Skin-Color Measurements}

Results are presented in Table 2. Mean erythema indices ranged from 149.6 (SD 43.4) at the UIA of the young group to 233.7 (SD 55.4) at the DFA of the midaged subjects. Correlations with age ranged between 0.01 and 0.18 . Average melanin indices were lowest at the UIA of the midaged group with 129.8 (SD 23.3) and highest at the DFA of the old group with 177.1 (SD 36.7). Correlation coefficients ranged between 0.14 and 0.27 . Mean $L *$ decreased with increasing age at all areas, e.g., from 68.68 (SD 2.0), to 67.35 (SD 3.4), and to 65.59 (SD 1.74) at the VFA. The negative associations with age ranged from -0.30 at the UIA to -0.44 at the VFA. Mean redness $(a *)$ varied between 4.95 (SD 1.84) and 7.58 (SD 1.57) as measured on the UIA of the midaged and DFA of the older subjects, respectively. Means among age groups were comparable and associations with age were between 0.25 and 0.28 . Yellowness $(b *)$ values did not differ among age groups and correlations with age were between 0.08 and 0.19 . There were no statistically significant differences between age groups for any parameter.

Results of skin-color measurements were lowest at the UIA and highest at the DFA within the age groups for almost every parameter, e.g., $a * 4.95$ (SD 1.84), 5.50 (SD 1.86), and 7.11 (SD 1.61) at the UIA, VFA, and DFA of the midaged subjects, respectively. The opposite was observed for $L *$, e.g., 69.21 (SD 2.17), 68.68 (SD 2.00), and 65.89 (SD 2.27) at the UIA, VFA, and DFA of the young group.

\subsection{Quantification of Dyspigmentation}

Results are presented in Table 2. Hyperpigmentation indices increased with age, e.g., 0.06 (SD 0.01), 0.09 (SD 0.03, and 0.12 (SD 0.04) at the VFA of the young, midaged, and older subjects. Means were statistically significantly different at all skin areas. Correlation coefficients of hyperpigmentation indices and age were between 0.61 and 0.74 . Hypopigmentation indices ranged from 0.00 to 0.14 and were significantly different between the age groups at all measurement fields. Correlations with age were between 0.676 and 0.740 . Overall, pigmentation intensity decreased with increasing age, e.g., -2.43 (SD 0.50 ), -2.73 (SD 0.86), and -3.27 (SD 0.77) at the VFA. Differences between the age groups were statistically not significant. The strongest association with age was found on the VFA, $r=-0.48$.

\subsection{Differences Between Skin Areas}

Results are presented in Table 3. Erythema indices differed significantly between all measurement fields $(p<0.001)$. The mean difference was highest between the DFA and the UIA. Mean melanin indices differed statistically significantly between the DFA-UIA and the DFA-VFA $(p<0.001)$. Luminance was significantly different between all the measurement fields and mean differences ranged from -3.57 to -1.18 . Redness $(a *)$ and yellowness $(b *)$ were significantly different when comparing the DFA to the other measurement fields, $p<0.001$ in all cases. Hyper- and hypopigmentation indices were comparable between the VFA and the UIA and statistically significantly different when comparing DFA with the other areas $(p<0.001)$. Overall pigmentation intensity was statistically different between the DFA and the UIA $(p<0.001)$ and between the DFA with the VFA $(p<0.001)$.

\subsection{Reliability of Measurements}

Results are shown in Table 2. Both erythema and melanin indices revealed good reliability between the three repeated measurements as indicated by the ICCs higher than 0.8. $L * a *$, and $b *$ values revealed ICCs of at least 0.85 at all skin areas and showed excellent reliability of $L *$ among the three repeated measurements. Dyspigmentation parameters based on image processing had good to excellent reliability. ICC values of the hyperpigmentation index were above 0.81 and reliability estimates of hypopigmentation index were greater than 0.91 . Overall, pigmentation intensity at the DFA had the lowest ICC coefficients of 0.8 .

\section{Discussion}

In this study, we showed changes in skin pigmentation during aging on sun-exposed and sun-protected areas. We demonstrated 
Table 2 Means, standard deviations, associations, and reliability coefficients of skin color and dyspigmentation parameters.

\begin{tabular}{|c|c|c|c|c|c|c|c|c|}
\hline & $\begin{array}{l}\text { Young group } \\
\quad(n=8)\end{array}$ & $\begin{array}{l}\text { Midaged group } \\
\quad(n=8)\end{array}$ & $\begin{array}{l}\text { Old group } \\
(n=8)\end{array}$ & $\begin{array}{c}\text { Total } \\
(n=24)\end{array}$ & $p$ value ${ }^{a}$ & $\begin{array}{l}\text { Corre-lation } \\
\text { with age }{ }^{\mathrm{b}}\end{array}$ & $\begin{array}{c}\text { ICC } \\
(n=24)\end{array}$ & $\begin{array}{l}95 \% \mathrm{Cl} \\
\text { range }\end{array}$ \\
\hline \multicolumn{9}{|c|}{ Skin color measurements } \\
\hline \multicolumn{9}{|c|}{ Erythema index } \\
\hline UIA & $149.6(43.4)$ & $155.3(44.0)$ & $175.5(62.5)$ & $160.1(49.8)$ & 0.570 & 0.181 & 0.922 & $0.854-0.963$ \\
\hline VFA & $182.4(39.6)$ & $198.5(56.3)$ & $191.3(48.9)$ & $190.8(47.1)$ & 0.806 & 0.014 & 0.813 & $0.672-0.907$ \\
\hline DFA & $214.0(44.1)$ & $233.7(55.4)$ & $221.5(59.8)$ & $223.1(51.8)$ & 0.760 & 0.033 & 0.904 & $0.822-0.954$ \\
\hline \multicolumn{9}{|c|}{ Melanin index } \\
\hline UIA & $136.6(24.5)$ & $129.7(23.3)$ & $147.1(33.1)$ & $137.8(27.1)$ & 0.455 & 0.145 & 0.929 & $0.867-0.966$ \\
\hline VFA & $133.3(30.3)$ & $133.7(19.8)$ & $151.1(26.1)$ & $139.4(26.0)$ & 0.307 & 0.270 & 0.901 & $0.816-0.952$ \\
\hline DFA & $165.7(37.7)$ & $159.3(33.5)$ & $177.1(36.7)$ & $167.3(35.2)$ & 0.613 & 0.147 & 0.939 & $0.885-0.971$ \\
\hline \multicolumn{9}{|l|}{$L *$} \\
\hline UIA & $69.21(2.17)$ & $69.07(3.02)$ & $66.87(2.85)$ & $68.38(2.81)$ & 0.176 & -0.302 & 0.952 & $0.908-0.977$ \\
\hline VFA & $68.68(2.00)$ & $67.35(3.40)$ & $65.59(1.74)$ & $67.21(2.71)$ & 0.065 & -0.444 & 0.917 & $0.844-0.960$ \\
\hline DFA & $65.89(2.27)$ & $64.49(3.15)$ & $63.52(3.14)$ & $64.64(2.93)$ & 0.276 & -0.330 & 0.916 & $0.844-0.960$ \\
\hline \multicolumn{9}{|l|}{$a *$} \\
\hline UIA & $5.08(0.69)$ & $4.95(1.84)$ & $5.99(1.41)$ & $5.34(1.42)$ & 0.290 & 0.257 & 0.923 & $0.855-0.963$ \\
\hline VFA & $5.15(0.64)$ & $5.50(1.86)$ & $6.12(1.42)$ & $5.59(1.40)$ & 0.393 & 0.281 & 0.897 & $0.810-0.950$ \\
\hline DFA & $6.78(0.86)$ & $7.11(1.61)$ & $7.58(1.57)$ & $7.16(1.37)$ & 0.530 & 0.247 & 0.891 & $0.799-0.947$ \\
\hline \multicolumn{9}{|l|}{$b *$} \\
\hline UIA & $15.32(1.60)$ & $14.52(2.29)$ & $16.07(2.01)$ & $15.30(2.00)$ & 0.314 & 0.165 & 0.847 & $0.726-0.925$ \\
\hline VFA & $15.02(1.37)$ & $14.70(2.07)$ & $15.62(1.84)$ & $15.11(1.75)$ & 0.588 & 0.192 & 0.925 & $0.858-0.964$ \\
\hline DFA & $18.03(1.55)$ & $17.78(2.19)$ & $18.16(1.94)$ & 17.99 (1.83) & 0.922 & 0.077 & 0.905 & $0.824-0.954$ \\
\hline
\end{tabular}

Dyspigmentation measurements

Hyperpigmentation index

$\begin{array}{llllllllr}\text { UIA } & 0.07(0.02) & 0.09(0.04) & 0.13(0.04) & 0.10(0.04) & \mathbf{0 . 0 0 9} & \mathbf{0 . 6 1 1} & \mathbf{0 . 9 1 9} & 0.822-0.964 \\ \text { VFA } & 0.06(0.01) & 0.09(0.03) & 0.12(0.04) & 0.09(0.04) & \mathbf{0 . 0 0 1} & \mathbf{0 . 7 3 9} & \mathbf{0 . 9 3 2} & 0.850-0.970 \\ \text { DFA } & 0.09(0.04) & 0.17(0.04) & 0.18(0.04) & 0.15(0.06) & \mathbf{0 . 0 0 1} & \mathbf{0 . 7 1 2} & 0.812 & 0.614-0.914\end{array}$

Hypopigmentation index

\begin{tabular}{|c|c|c|c|c|c|c|c|c|}
\hline UIA & $0.01(0.01)$ & $0.02(0.01)$ & $0.07(0.05)$ & $0.03(0.04)$ & 0.001 & 0.676 & 0.943 & $0.872-0.975$ \\
\hline VFA & $0.00(0.01)$ & $0.03(0.03)$ & $0.07(0.04)$ & $0.03(0.04)$ & $<0.001$ & 0.740 & 0.950 & $0.889-0.978$ \\
\hline DFA & $0.04(0.03)$ & $0.12(0.05)$ & $0.14(0.05)$ & $0.10(0.06)$ & $<0.001$ & 0.727 & 0.910 & $0.803-0.960$ \\
\hline \multicolumn{9}{|c|}{ Overall pigmentation intensity } \\
\hline UIA & $-2.57(0.53)$ & $-2.51(0.78)$ & $-2.97(0.76)$ & $-2.68(0.70)$ & 0.370 & -0.252 & 0.954 & $0.897-0.980$ \\
\hline VFA & $-2.43(0.50)$ & $-2.73(0.86)$ & $-3.27(0.77)$ & $-2.81(0.78)$ & 0.083 & -0.481 & 0.984 & $0.964-0.993$ \\
\hline DFA & $-3.35(0.76)$ & $-3.71(0.75)$ & $-4.00(0.83)$ & $-3.69(0.80)$ & 0.277 & -0.361 & 0.803 & $0.597-0.910$ \\
\hline
\end{tabular}

Note: ICC, intraclass correlation coefficient; Cl, confidence interval; UIA, upper inner arm; VFA, volar forearm; DFA, dorsal forearm; bold, statistically significant difference, correlation $>0.3, \mathrm{ICC}>0.9$.

${ }^{a} A N O V A$ between age groups.

bPearson's correlation with age; 
Table 3 Paired comparisons between skin areas.

\begin{tabular}{|c|c|c|c|c|}
\hline \multirow{2}{*}{$\begin{array}{l}\text { Measurement } \\
\text { Comparison of skin sites }\end{array}$} & \multirow[b]{2}{*}{$\begin{array}{c}\text { Mean } \\
\text { difference }\end{array}$} & \multicolumn{2}{|c|}{$95 \% \mathrm{Cl}$} & \multirow[b]{2}{*}{$p^{a}$} \\
\hline & & $\begin{array}{l}\text { Lower } \\
\text { bound }\end{array}$ & $\begin{array}{l}\text { Upper } \\
\text { bound }\end{array}$ & \\
\hline \multicolumn{5}{|l|}{ Erythema index } \\
\hline VFA-UIA & 30.61 & 16.41 & 44.80 & $<0.001$ \\
\hline DFA-UIA & 62.91 & 48.79 & 77.04 & $<0.001$ \\
\hline DFA-VFA & 32.31 & 19.60 & 15.01 & $<0.001$ \\
\hline \multicolumn{5}{|l|}{ Melanin index } \\
\hline VFA-UIA & 1.58 & -5.21 & 8.38 & 0.635 \\
\hline DFA-UIA & 29.53 & 17.01 & 42.04 & $<0.001$ \\
\hline DFA-VFA & 27.94 & 18.55 & 37.34 & $<0.001$ \\
\hline
\end{tabular}

Chromameter $L *$

$\begin{array}{lllll}\text { VFA-UIA } & -1.18 & -1.79 & -0.56 & \mathbf{0 . 0 0 1} \\ \text { DFA-UIA } & -3.75 & -4.81 & -2.69 & <\mathbf{0 . 0 0 1} \\ \text { DFA-VFA } & -2.57 & -3.33 & -1.82 & <0.001\end{array}$

Chromameter $a *$

$\begin{array}{lllll}\text { VFA-UIA } & 0.25 & -0.02 & 0.52 & 0.066 \\ \text { DFA-UIA } & 1.81 & 1.44 & 2.18 & <0.001 \\ \text { DFA-VFA } & 1.56 & 1.26 & 1.86 & <0.001\end{array}$

Chromameter $b *$

$\begin{array}{lcccc}\text { VFA-UIA } & -0.19 & -0.81 & 0.43 & 0.528 \\ \text { DFA-UIA } & 2.69 & 1.89 & 3.49 & <0.001 \\ \text { DFA-VFA } & 2.88 & 2.39 & 3.37 & <\mathbf{0 . 0 0 1}\end{array}$

Hyperpigmentation index

$\begin{array}{lllll}\text { VFA-UIA } & -0.008 & -0.021 & 0.005 & 0.194 \\ \text { DFA-UIA } & 0.052 & 0.033 & 0.071 & <\mathbf{0 . 0 0 1} \\ \text { DFA-VFA } & 0.061 & 0.048 & 0.073 & <0.001\end{array}$

Hypopigmentation index

\begin{tabular}{lcccc} 
VFA-UIA & -0.001 & -0.009 & 0.007 & 0.787 \\
DFA-UIA & 0.066 & 0.047 & 0.086 & $<\mathbf{0 . 0 0 1}$ \\
DFA-VFA & 0.068 & 0.051 & 0.085 & $<\mathbf{0 . 0 0 1}$ \\
verall pigmentation intensity & & & & \\
VFA-UIA & -0.129 & -0.291 & 0.034 & 0.116 \\
DFA-UIA & -1.008 & -1.268 & -0.748 & $<\mathbf{0 . 0 0 1}$ \\
DFA-VFA & -0.879 & -1.074 & -0.684 & $<\mathbf{0 . 0 0 1}$ \\
\hline
\end{tabular}

Note: $\mathrm{Cl}$, confidence interval; UIA, upper inner arm; VFA, volar forearm; DFA, dorsal forearm; bold, statistically significant.

${ }^{a}$ Statistical significance of paired sample $t$-test. an increase in pigmentary heterogeneity in extrinsically and intrinsically aged skin. The color of the sun-exposed forearm skin was found to differ from photoprotected areas already in the younger adults.

Estimates by the Mexameter device are sparsely available from comparable skin areas. Similar melanin and erythema indices were reported of the VFA, ${ }^{28}$ and Korean authors also found no associations of these indices with age at various skin areas including the measurement fields of our study. ${ }^{33}$ In the current study, the sun-exposed DFA was found to be darker than "intermediate" VFA that was darker than the photo-protected UIA. This was observed by all color parameters for the whole sample and also per age group. Similar differences in skin color between intrinsic- and extrinsic-aged skin were demonstrated in previous studies by other measurement techniques. ${ }^{25,34,35}$ Freis et al. ${ }^{12}$ described comparable relationships between luminance and age on both sun-exposed and photo-protected skins in a large sample. The differences between the VFA and the UIA regarding the redness parameters erythema index and $a *$ may probably be explained by a high sensitivity of vascularization to actinic damage. ${ }^{36}$

An age-dependent increase of dyspigmentation at sunexposed skin areas is supported by clinical observations. ${ }^{15-17,37}$ Recent studies provided evidence of increasing heterogeneity in cutaneous pigment distribution by various methods of image processing. ${ }^{17,18,38,39}$ The definition of extrinsic aging already includes the presence of dyspigmentation, ${ }^{3,4}$ but for the first time we provide empirical evidence of an age-dependent increase of pigmentary heterogeneity also at sun-protected skin areas. The DFA clearly displayed the highest degree of dyspigmentation, which is in accordance with clinical observations and mechanistic reasoning.

For better understanding of photo-carcinogenesis, studies of cutaneous sun damage are needed. ${ }^{22}$ These investigations are frequently based on the comparison of extrinsic- and intrinsic-aged skin to avoid variations between subjects. ${ }^{23}$ Previous studies made these comparisons by comparing facial skin with areas as the UIA or the lower back (buttock). ${ }^{34,35}$ Because of a high-anatomic comparability of the skin areas, the DFA served as photodamaged skin and the VFA and UIA as a surrogate of intrinsic-aged skin in the current study. Based on the differences in $L *$ and erythema indices among the investigational areas, one might argue whether the VFA is really the best reference for studying intrinsic aging. Our results clearly support UVdamage of the VFA that increases with age. Therefore, we propose the UIA to be used as a model skin area for studying primarily intrinsic aging in future studies. The UIA as an intrinsic comparison might additionally be easier to study and be closer to real-life conditions. However, differences between the UIA, VFA, and DFA in skin anatomy and physiology still have to be kept in mind when planning studies using the forearms, e.g., there are more vellus-type hair follicles at the dorsal side.

Skin-color measurements and dyspigmentation estimates were found to have a high reliability. ICC coefficients were greater than 0.8 for all parameters at all measurement fields, indicating a good reproducibility within studies. ${ }^{31,32}$ Additionally, the lower bounds of the $95 \%$ confidence interval were above 0.8 for the melanin index, $L * a *$, and hypopigmentation index and were greater than 0.6 for all the other pigmentation parameters. Previous trials showed the validity of color measurements ${ }^{28,40}$ and parameters of dyspigmentation. ${ }^{17}$ 
(a)
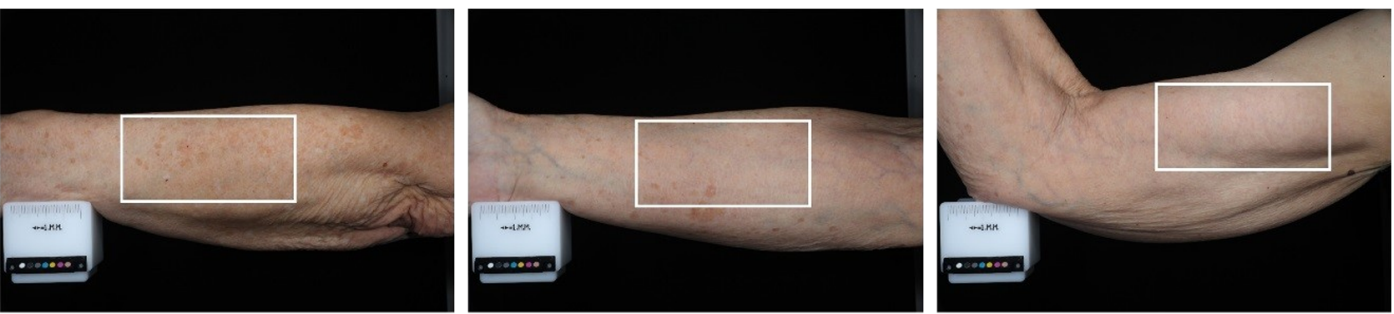

(b)
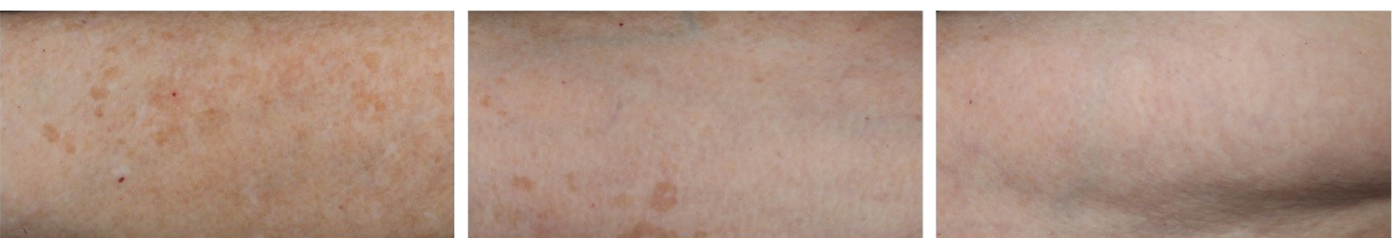

(c)
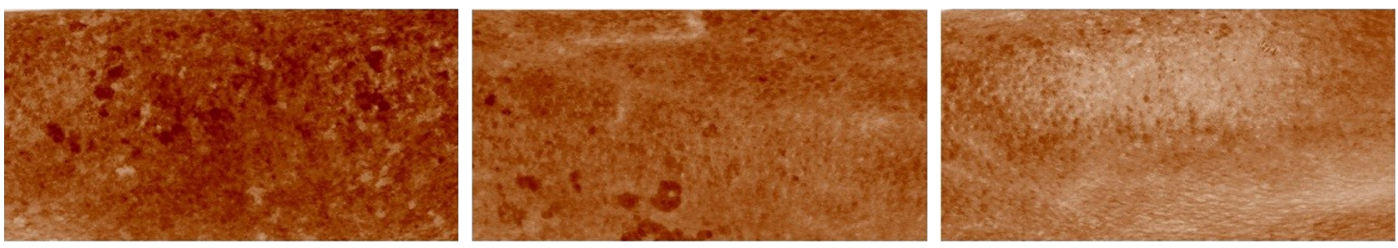

(d)
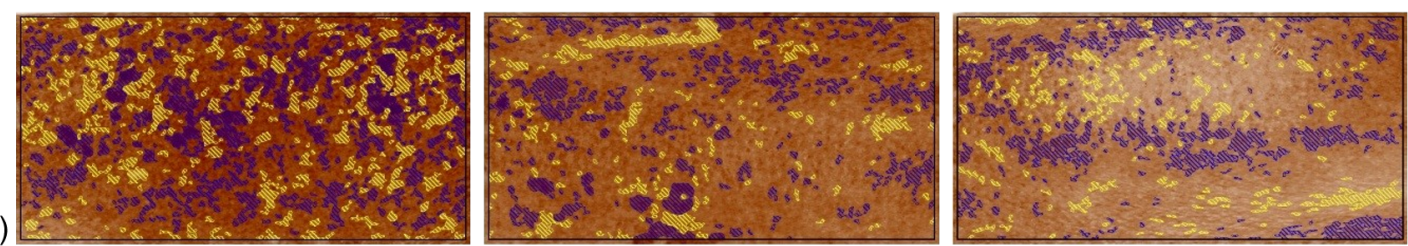

Fig. 1 Measurement of skin dyspigmentation on DFA, VFA, and the UIA by image processing. (a) As captured, crosspolarized clinical images, with the measurement area outlined in white. (b) Images cropped to the measurement area. (c) $\mathrm{RBX}^{\circledR}$-Brown images of the measurement area. (d) Hyperpigmentation (blue) and hypopigmentation (yellow) detection inside the arm measurement areas from $\mathrm{RBX}^{\circledR}$-Brown image analysis.

In conclusion, we demonstrate an increase in hyperpigmentation and hypopigmentation at both intrinsically and extrinsically aged skin areas, which is evident in young adulthood already. Associations with chronological age and dyspigmentation estimates were higher compared to the color measurements. Therefore, dyspigmentation parameters seem to be better biomarkers for UV damage than overall color measurements. Irrespectively, all color and dyspigmentation estimates showed high reliability supporting their use in clinical research. The UIA as a sun-protected area should be preferred when studying intrinsic aging compared to the VFA skin where extrinsic and intrinsic aging is present.

\section{Limitations}

In order to reduce a possible bias due to gender, we included females only. The sample of 24 subjects was rather small because of the explorative character of this study.

\section{Acknowledgments}

The authors acknowledge the support of the C. Richter and $\mathrm{H}$. Wehrmeyer in the conduct of the study and data management and thank all the subjects for participating in this study. G. Dobos was a recipient of the Hans Schaefer Young Researcher Grant. www.hairskinberlin.com

\section{References}

1. R. J. Hay et al., "The global burden of skin disease in 2010: an analysis of the prevalence and impact of skin conditions," J. Invest. Dermatol. 134(6), 1527-1534 (2014).

2. M. Yaar and B. A. Gilchrest, "Photoageing: mechanism, prevention and therapy," Br. J. Dermatol. 157(5), 874-887 (2007).

3. J. H. Epstein, "Photocarcinogenesis, skin cancer, and aging," J. Am. Acad. Dermatol. 9(4), 487-502 (1983).

4. B. A. Gilchrest, "Skin aging and photoaging: an overview," J. Am. Acad. Dermatol. 21, 610-613 (1989).

5. G. Dobos et al., "Evaluation of skin ageing: a systematic review of clinical scales," Br. J. Dermatol. 172(5), 1249-1261 (2015).

6. G. E. Pierard et al., "Pigmentary changes in skin senescence," J. Appl. Cosmetol. 9, 57-63 (1991).

7. S. Del Bino et al., "Chemical analysis of constitutive pigmentation of human epidermis reveals constant eumelanin to pheomelanin ratio," Pigm. Cell Melanoma Res. 28(6), 707-717 (2015).

8. J. P. Ortonne, "Pigmentary changes of the ageing skin," Br. J. Dermatol. 122(Suppl. 35), 21-28 (1990).

9. J. P. Ortonne and D. L. Bissett, "Latest insights into skin hyperpigmentation," J. Investig. Dermatol. Symp. Proc. 13(1), 10-14 (2008).

10. J. Ayer et al., "A comparison of atrophic and hypertrophic facial photoageing," J Invest. Dermatol. Symp Proc. 135(Suppl. 2), 216 (2015).

11. Y. R. Helfrich et al., "Clinical, histologic, and molecular analysis of differences between erythematotelangiectatic rosacea and telangiectatic photoaging," JAMA Dermatol. 151(8), 825-836 (2015).

12. O. Freis, G. Perie, and A. Rathjens, "Skin's mechanical and optical properties," Cosmet. Toiletries 129(4), 66-75 (2014).

13. C. Galzote et al., "Characterization of facial skin of various Asian populations through visual and non-invasive instrumental evaluations: influence of seasons," Skin Res. Technol. 20(4), 453-462 (2014). 
14. C. Trojahn et al., "Characterizing facial skin ageing in humans: disentangling extrinsic from intrinsic biological phenomena," BioMed Res. Int. 2015, 318586 (2015).

15. K. Ezzedine et al., "Freckles and solar lentigines have different risk factors in Caucasian women," J. Eur. Acad. Dermatol. Venereol. 27(3), e345-e356 (2013).

16. F. Flament et al., "Effect of the sun on visible clinical signs of aging in Caucasian skin," Clin. Cosmet. Investig. Dermatol. 6, 221-232 (2013).

17. G. Dobos et al., "Quantifying dyspigmentation in facial skin ageing: an explorative study," Int. J. Cosmet. Sci. 37(5), 542-549 (2015).

18. P. J. Matts et al., "Color homogeneity and visual perception of age, health, and attractiveness of female facial skin," J. Am. Acad. Dermatol. 57(6), 977-984 (2007).

19. J. H. Chung et al., "Cutaneous photodamage in Koreans," Arch. Dermatol. 137, 1043-1051 (2001).

20. P. Aguilera et al., "Prevalence study of nevi in children from Barcelona, dermoscopy, constitutional and environmental factors," Dermatology 218(3), 203-214 (2009).

21. J. Wendt et al., "Site-dependent actinic skin damage as risk factor for melanoma in a central European population," Pigm. Cell Melanoma Res. 25(2), 234-242 (2012).

22. J. Lock-Andersen, K. T. Drzewiecki, and H. C. Wulf, "The measurement of constitutive and facultative skin pigmentation and estimation of sun exposure in caucasians with basal cell carcinoma and cutaneous malignant melanoma," Br. J. Dermatol. 139, 610-617 (1998).

23. J. Bhawan et al., "Photoaging versus intrinsic aging: a morphologic assessment of facial skin," J. Cutan. Pathol. 22, 154-159 (1995).

24. C. Edwards, R. Heggie, and R. Marks, "A study of differences in surface roughness between sun-exposed and unexposed skin with age," Photodermatol. Photoimmunol. Photomed. 19(4), 169-174 (2003).

25. S. Alaluf et al., "Ethnic variation in melanin content and composition in photoexposed and photoprotected human skin," Pigm. Cell Melanoma Res. 15, 112-118 (2002).

26. J. Blaak et al., "Irritability of the skin barrier: a comparison of chronologically aged and photo-aged skin in elderly and young adults," Eur. Geriatr. Med. 2, 208-211 (2011).

27. M. A. Farage et al., "Intrinsic and extrinsic factors in skin ageing: a review," Int. J. Cosmet. Sci. 30, 87-95 (2008).

28. M. Baquie and B. Kasraee, "Discrimination between cutaneous pigmentation and erythema: comparison of the skin colorimeters Dermacatch and Mexameter," Skin Res. Technol. 20(2), 218-227 (2014).

29. G. E. Pierard, "EEMCO guidance for the assessment of skin colour," J. Eur. Acad. Dematol. Venereol. 10, 1-11 (1998).

30. R. Demirli et al., "RBX technology overview. White paper," 2007, www .canfieldsci.com (13 June 2016).

31. J. Kottner et al., "Guidelines for reporting reliability and agreement studies (GRRAS) were proposed," Int. J. Nurs. Stud. 48(6), 661-671 (2011).

32. C. B. Terwee et al., "Quality criteria were proposed for measurement properties of health status questionnaires," J. Clin. Epidemiol. 60(1), 34-42 (2007).

33. K. Y. Roh et al., "Pigmentation in Koreans: study of the differences from caucasians in age, gender and seasonal variations," Br. J. Dermatol. 144, 94-99 (2001).

34. P. Rubegni et al., "Quantitative characterization and study of the relationship between constitutive and facultative skin color and phototype in caucasians," Photochem. Photobiol. 70(3), 303-307 (1999).

35. T. H. Wong, I. J. Jackson, and J. L. Rees, "The physiological and phenotypic determinants of human tanning measured as change in skin colour following a single dose of ultraviolet B radiation," Exp. Dermatol. 19(7), 667-673 (2010).

36. J. H. Chung and H. C. Eun, "Angiogenesis in skin aging and photoaging," J. Dermatol. 34, 593-600 (2007).

37. E. Tschachler and F. Morizot, "Ethnic differences in skin aging," in Textbook of Aging Skin, M. A. Farage, K. W. Miller, and H. I. Maibach, Eds., Vol. 1, pp. 23-31, Springer, Berlin, Heidelberg (2010).
38. J de Rigal et al., "The effect of age on skin color and color heterogeneity in four ethnic groups," Skin Res. Technol. 16(2), 168-178 (2010).

39. G. G. Hillebrand, "Quantitative evaluation of skin condition in an epidemiological survey of females living in northern versus southern Japan," J. Dermatol. Sci. 27(Suppl. 1), 42-52 (2001).

40. H. Ohshima et al., "Melanin and facial fluorescence as a marker of yellowish discoloration with aging," Skin Res. Technol. 15(4), 496-502 (2009).

Gabor Dobos received his degree in medicine from the Semmelweis University of Budapest in 2012. In 2016 he finshed his PhD at the Clinical Research Center for Hair and Skin Science at the Department of Dermatology and Allergy at the Charité-Universitätsmedizin Berlin. His research focuses on skin diseases of the elderly, validation of measurement techniques, and clinical scores in the context of skin ageing.

Carina Trojahn received her diploma in human biology from the University of Marburg in 2010 and worked as a project manager of clinical studies in the field of dermatology and cosmetic science. In 2016, she completed her PhD at the Clinical Research Center for Hair and Skin Science at the Department of Dermatology and Allergy at the Charité-Universitätsmedizin Berlin. Her research focuses on validation of methods for measuring structural and functional changes during skin aging.

Brian D'Alessandro is a research scientist at Canfield Scientific Inc., New Jersey, United States. He received his PhD in 2012 from the New Jersey Institute of Technology (NJIT). His research focuses on developing unique image analysis and computer vision algorithms for skin feature detection and measurement, skin lesion analysis, and other cosmetic and clinical applications.

Sachin Patwardhan is a principal scientist at Canfield Scientific Inc., New Jersey, United States. He received his PhD in 2003 from New Jersey Institute of Technology (NJIT). Earlier, he was a research associate in the Department of Radiology, Washington University School of Medicine, Saint Louis, Missouri. His research interests are in developing optical imaging and image analysis solutions for clinical and preclinical research in dermatology, plastic surgery and skin aesthetic applications.

Douglas Canfield is the founder and president of Canfield Scientific Inc., and vice president of Laennec Publishing Inc. He also serves as an adjunct clinical professor, Department of Medicine, Georgetown University Medical Center and director for World Cranialfacial Foundation, Dallas, Texas. Canfield Scientific specializes in photographic documentation, and developing 2D and 3D imaging solutions and protocols for clinical trials to demonstrate drug efficacy. It also provides specialized clinical imaging solutions for dermatologists and plastic surgeons.

Ulrike Blume-Peytavi is a full university professor and the executive medical director of the Department of Dermatology and Allergy at the Charité-Universitätsmedizin Berlin. She is the director of the Clinical Research Center for Hair and Skin Science and of the pediatric dermatology outpatient section. Her research focuses on skin and hair physiology over the life course, skin care regimen, pediatric dermatology, and follicular targeting.

Jan Kottner is the scientific director of the Clinical Research Center for Hair and Skin Science at the Department of Dermatology and Allergy at the Charité-Universitätsmedizin Berlin. He is an executive board member of the EPUAP. His research interests are skin barrier maintenance, protection, restoration, and skin problems and skin care interventions in aged and care-dependent subjects. He also has special expertise in the development and validation of clinical diagnoses, classifications, and scores. 